ORIGINAL PAPER

\title{
L1 CELL ADHESION MOLECULE (L1CAM) AND NERVE GROWTH FACTOR RECEPTOR (NGFR, P75) EXPRESSION PATTERNS IN SOLID PSEUDOPAPILLARY NEOPLASM OF THE PANCREAS
}

\author{
ŁUKASZ LISZKA
}

Department of Pathomorphology and Molecular Diagnostics, Medical University of Silesia, Katowice, Poland

\begin{abstract}
Solid pseudopapillary neoplasm (SPN) is a pancreatic tumor, which should be distinguished from neuroendocrine tumors (NET). It was postulated that SPN arise from the neural crest (NC). The purpose of the study was to examine expression levels of NC markers: L1 cell adhesion molecule (L1CAM) and nerve growth factor receptor (NGFR) in SPN and NET using immunohistochemistry (IHC) and tissue microarrays, aiming to test their potential utility as auxiliary IHC markers for differential diagnosis of SPN vs. NET. In the training cohort $(n=16$ SPN), all cases showed L1CAM expression (usually weak, median extent $45 \%$ of cells), and NGFR expression (usually moderate to strong, median extent $100 \%$ of cells). In the validation cohort ( $\mathrm{n}=10 \mathrm{SPN}$ ), $90 \%$ of cases were L1CAM-positive (usually weak expression, median extent $15 \%$ of cells), and $100 \%$ were NGFR-positive (usually weak expression, median extent $70 \%$ of cells). Among NET cases $(\mathrm{n}=29)$ L1CAM was found in $2(7 \%)$, and NGFR in 1 case (3\%). L1CAM and NGFR were expressed in SPN, but the intensities and extent of IHC staining differed across the cases. L1CAM and NGFR expression was rare in NET. Both markers may be further tested for their diagnostic utility for SPN vs. NET differential diagnosis. L1CAM/NGFR expression supports NC origin/differentiation of SPN.
\end{abstract}

Key words: pancreas, pancreatic neoplasms, neural cell adhesion molecule L1, nerve growth factor receptor.

\section{Introduction}

Solid pseudopapillary neoplasm (SPN) is a lowgrade malignant pancreatic tumor of uncertain histogenesis, composed of discohesive cells arranged in pseudopapillae [1]. SPN usually develops in young women [1]. Metastatic disease was reported in 5-15\% of patients with SPN, but tumor-related mortality was exceptional $[1,2]$. Gain-of-function $\beta$-catenin (CTNNB1) gene mutation resulting in activation of the Wnt pathway is a characteristic molecular alteration in SPN [3]. The diagnostic immunohistochemical (IHC) hallmark of SPN is nuclear expression of $\beta$-catenin, which is caused by translocation of the altered protein to the nucleus [3]. The cellular differentiation, as well as genomic, transcriptomic, and proteomic profile of SPN differs from pancreatic ductal adenocarcinomas and neuroendocrine neoplasms $[1,4,5,6,7,8,9,10]$.

SPN should be distinguished from other "solid-cellular" pancreatic tumors: neuroendocrine tumors (NET), acinar cell carcinomas, and pancreatoblastomas [1]. SPN may histopathologically resemble NET, and vice versa [11]. Microscopic features are sometimes inconclusive and therefore IHC markers may be helpful in the differential diagnosis $[11$, $12,13]$. Nuclear $\beta$-catenin, positive MME/CD10, dot-like CD99, and negative chromogranin A stains 
constitute a classical immunoprofile of SPN, which is found in NET only rarely $[1,11,13]$. New promising SPN markers are LEF1, TFE3, SOX10, SOX11 and AR [5, 14, 15]. The discriminatory value of classical and emerging IHC stains may be suboptimal [12], so new markers are needed.

L1 cell adhesion molecule/neural cell adhesion molecule L1 (L1CAM, N-CAM L1, CAML1, CD171, MIC5) is important for neural development, neuron adhesion/migration, axonal guidance, synaptogenesis, neurite growth/fasciculation, myelination, and cell survival [16]. It also has a role in cancer progression [16, 17]. L1CAM expression is an unfavorable prognostic factor in many malignancies [18].

Nerve growth factor receptor/low-affinity neurotrophin receptor/tumor necrosis factor receptor superfamily member 16 (NGFR, p75, p75NTR. TNFRSF16, CD271) is a $75 \mathrm{kDa}$ neurotrophin receptor responsible for development, growth, survival, and homeostasis of the nervous system [19, 20, 21, 22]. Development and progression of tumors may be modulated by NGFR [22]. NGFR acts as a tumor suppressor and marker of a subset of cancer stem cells $[21]$.

The neural crest (NC) is a group of migratory embryonal neuroectodermal cells, which transform into diverse types of tissue across the body [23]. There are similarities between processes of NC cell migration and cancer progression [24]. It was proposed that SPN may originate from NC cells - this hypothesis was based on the assessment of transcriptomic [5] and proteomic/IHC $[5,25]$ SPN profile. L1CAM and NGFR are markers of NC cells migrating to the gut [24, 26]. Moreover, L1CAM [27, 28, 29] and NGFR $[19,30]$ are expressed in tumors of neural/neuroectodermal/NC origin, as well as in some tumors of non-neural differentiation.

To the author's knowledge, presence of L1CAM and NGFR in SPN has not been hitherto investigated. Therefore, the objective of the study was to describe the IHC expression patterns of L1CAM and NGFR in SPN. In particular, the study aimed to verify whether these IHC markers may be useful for distinction of SPN vs. NET. SPN cases were also examined for expression levels of other neural/neuroendocrine/neuroectodermal/NC markers of potential diagnostic significance.

\section{Materials and methods}

\section{Study samples}

The study was based on in-house cases diagnosed in the author's institution in resection specimens. Two set of SPN were selected for the present study: (1) the training cohort - SPN diagnosed between
2014 and 2019,(2) the validation cohort - SPN diagnosed between 1985 and 2013, and described in detail in the previous papers $[2,31]$. For comparative purpose, 29 samples of NET of the pancreas examined in the previous study [31] were taken.

\section{Histopathological diagnoses and tissue preparation}

Diagnoses were confirmed based on World Health Organization histopathological criteria [1]. Specimens were formalin-fixed and routinely processed. All the cases were examined using tissue microarrays [2, 31]. Each SPN and NET case was represented by 3-4 cores (core diameter $1.5 \mathrm{~mm}$ ). Normal pancreatic tissue served for tissue/orientation control. The IHC stains performed in TMA included $\beta$-catenin, L1CAM, NGFR, $\beta$-1,3-glucuronyltransferase-1 (B3GAT1/CD57), chromogranin A (CHGA), enolase-2/neuron-specific enolase (ENO2/ NSE), neural cell adhesion molecule (NCAM/ CD56), GATA binding protein-3 (GATA3), glial fibrillary acidic protein (GFAP), neurofilament (NF), S100, and synaptophysin (SYP). Details of IHC assays and controls are described in Supplementary Data 1. Peripheral nerves were used as an internal tissue control for L1CAM [27] and NGFR [30] stains.

\section{IHC scoring}

IHC scoring was performed by a single investigator. Expression was examined in cell membrane/ cytoplasm/nucleus. Stain intensity was reported on a four-tier semi-quantitative scale $(0,1+, 2+, 3+)$ [27, 32]. Stain extent was estimated visually and documented as percentage of immuno-positive cells across all tissue cores [27, 32]. Histoscore values were obtained by multiplication of stain intensity scores and percentages of immuno-positive cells (range from 0 to 300) [32].

\section{Statistical analysis}

For correlations, Spearman's rank correlation coefficients were calculated. For proportions, Fisher's exact tests and standard measures of performance/ accuracy of diagnostic tests were used. Statistica 13 software (Tibco Software, Palo Alto, CA) and WinPepi [33] were utilized. Guidelines for reporting biomarker studies were followed [34].

\section{Ethics statement}

This observational study was performed with permission of the IRB without full review necessary for experimental studies. 

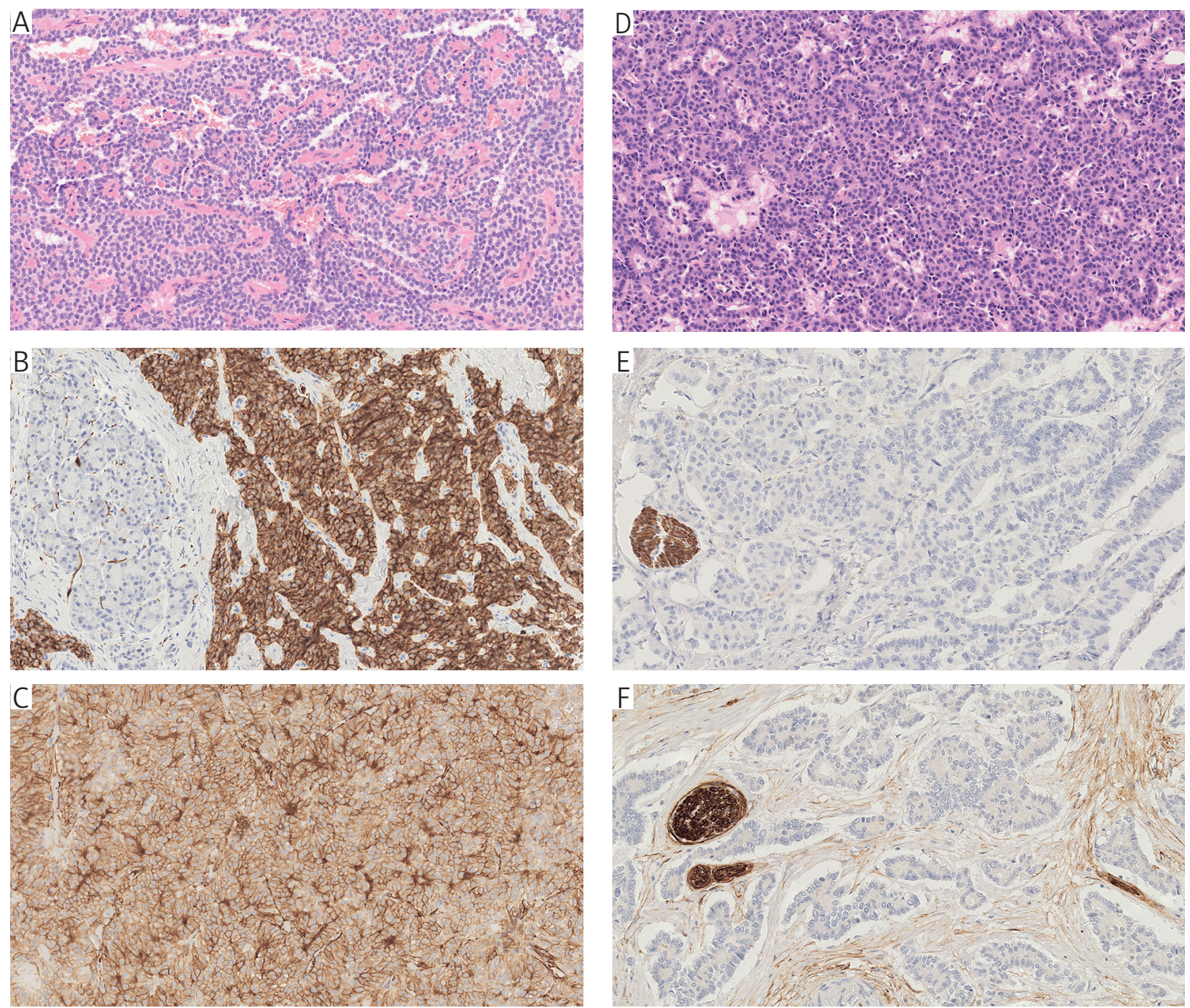

Fig. 1. A) Solid pseudopapillary neoplasm. Hematoxylin and eosin. Magnification; 40×. B) Strong membranous and cytoplasmic L1CAM expression in SPN. Periacinar L1CAM expression in normal pancreatic tissue (left). Magnification $40 \times$; C) Strong membranous and cytoplasmic NGFR expression in SPN. Magnification 40×; D) Neuroendocrine tumor of the pancreas. Hematoxylin and eosin. Magnification 40×; E) Negative L1CAM stain in NET. Nerve is strongly positive (left). Magnification $40 \times$; F) Negative NGFR stain in tumoral cells of NET. NGFR is expressed in a nerve (left) and in stromal cells. Magnification $40 \times$

\section{Results}

\section{Training cohort}

Seventeen SPN cases were available for the training cohort (Fig. 1A). One case was excluded due to loss of tumor tissue in TMA, leaving 16 cases for IHC assessment. A single case was represented by separate cores from primary and recurrent lesions. Clinico-pathological characteristics were concordant with the literature data $[1]$, and they are summarized in Supplementary Data 2. All cases showed abnormal (nuclear/cytoplasmic) $\beta$-catenin expression (not shown).

\section{Results of IHC examinations in the training cohort}

Results are summarized in Table I. L1CAM showed membranous expression with some cytoplasmic accentuation in all SPN cases, but stain intensity was heterogeneous across the tumors: weak, moderate, and strong (Fig. 1B) expression was detected in $81 \%$, $6 \%$, and $12 \%$ of tumors, respectively. Stain extent ranged from 5 to $100 \%$ (median $45 \%$ ). A single recurrent tumor showed slightly less intense staining in comparison to the primary tumor. Nerves detected in some SPN cores and in pancreatic parenchyma showed strong staining. Periacinar structures, possibly axons, showed moderate to strong expression in some cores; islets were negative or rarely weakly positive. Acini did not show evident expression. 


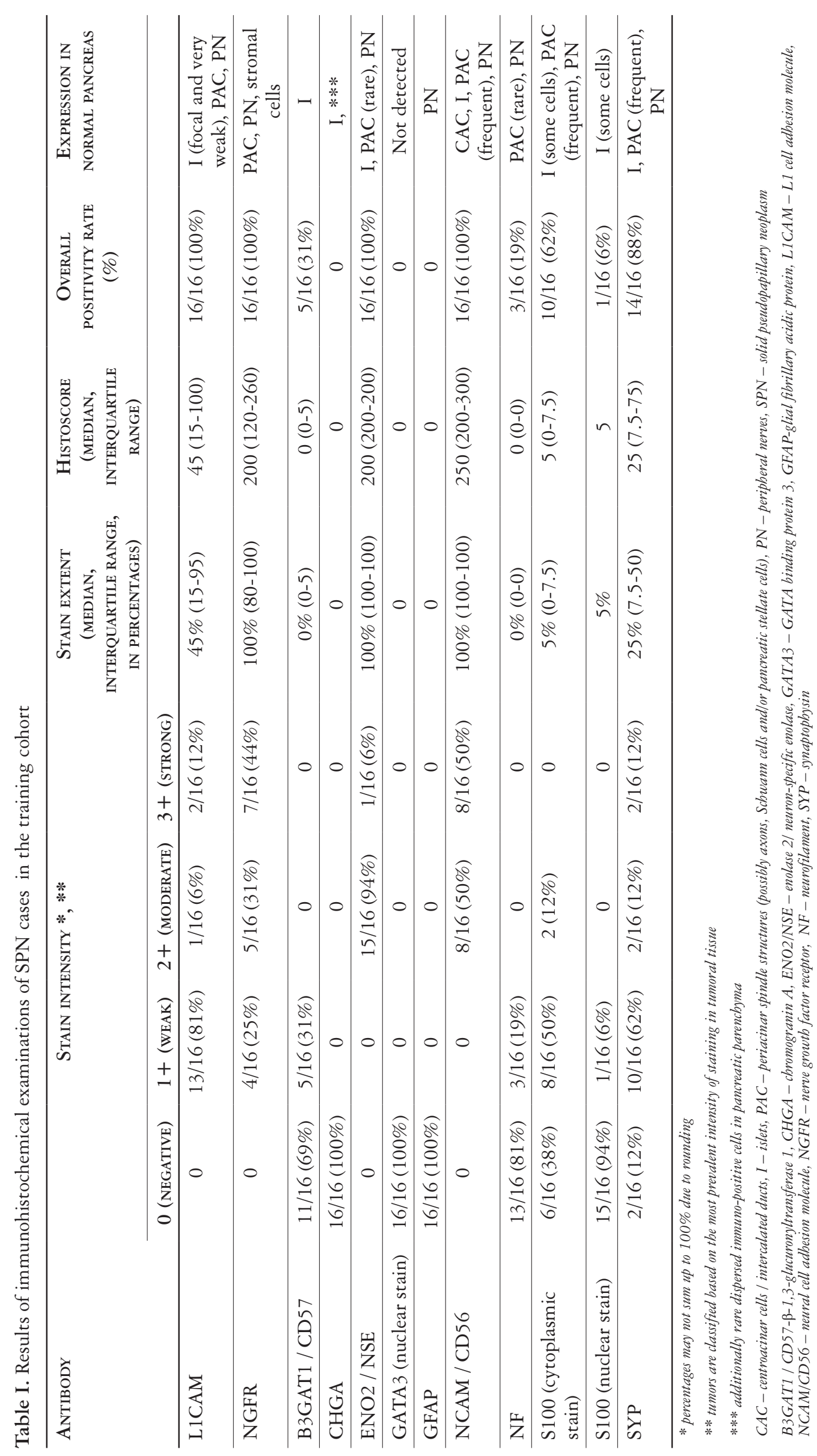



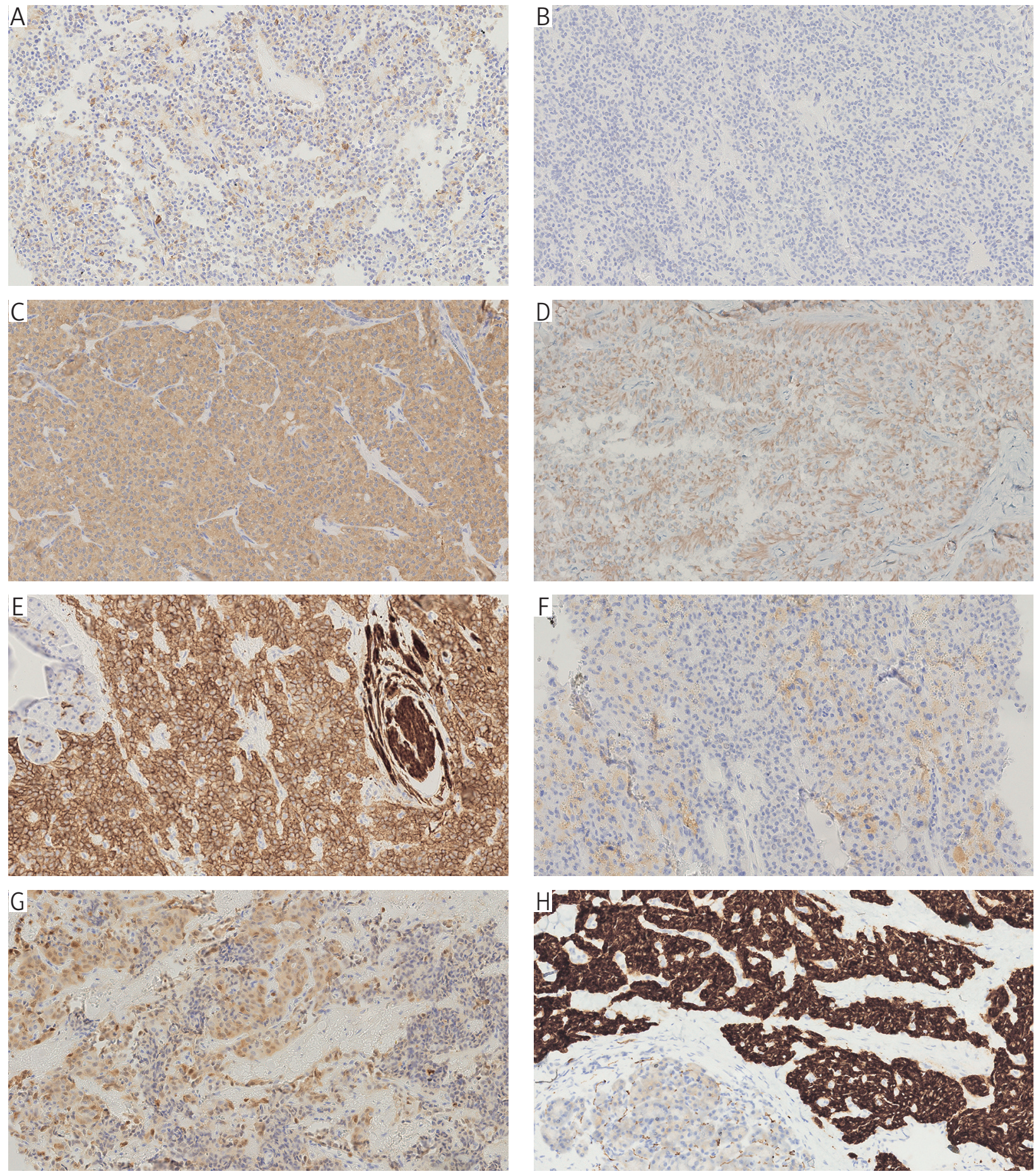

Fig. 2. A) Weak expression of B3GAT1/CD57 in SPN. Magnification 40×; B) All SPN cases were CHGA/chromogranin-A negative. Magnification 40×; C) ENO2/NSE expression in SPN. Magnification 40×; D) Weak cytoplasmic GATA3 expression in SPN. Magnification 40×; E) Membranous NCAM/CD56 expression in SPN. Nerve (right) and centroacinar compartment of normal pancreas (left) were also NCAM/CD56-positive. Magnification 40×; F) Weak NF expression in SPN. Magnification 40×; G) Cytoplasmic and nuclear S100 expression in SPN. Magnification 40×; $\mathrm{H})$ Strong SYP/synaptophysin expression in SPN. Normal pancreas is also seen at lower left. Magnification $40 \times$

Membranous and cytoplasmic NGFR expression was found in all SPN cases: weak, moderate, and strong (Fig. 1C) staining was found in $25 \%, 31 \%$, and $44 \%$ of cases, respectively. The extent of expression ranged from $70 \%$ to $100 \%$ with the exception of a single case, which showed expression in approximately $5 \%$ of cells. Expression in a single recurrent lesion was slightly less intense than in primary tumor. Stromal cells also expressed NGFR, in particular in the fibrovascular cores of pseudopapillae, in 
less cellular areas and at the periphery of the tumors. Peripheral nerves were strongly NGFR-positive. In normal pancreas NGFR was found in periacinar cells (strong expression), and in stromal periductal/perilobular cells (usually focal expression). Acinar cells and islets were non-reactive.

Cytoplasmic B3GAT1/CD57 was found in 5 (31\%) cases - it was usually weak and focal (from 5\% to $10 \%$ of cells, in a single case 40\%; Fig. 2A). Pancreatic islets showed weak staining, in particular in peripheral areas. All SPN were CHGA-negative (Fig. 2B). In normal pancreas immunoreactivity was found in islets and rare single dispersed cells in parenchyma. Cytoplasmic ENO2/NSE was detected in all SPN cases diffuse moderate or strong staining was present in 90$100 \%$ of cells (Fig. 2C); in rare cases dispersed areas with weak expression were also found. The recurrent tumor showed the same staining pattern as the primary lesion. ENO2/NSE was also positive in nerves, islets and rare scattered periacinar structures (possibly axons). Nuclear GATA3 was not found in any SPN sample. Weak/moderate (probably nonspecific) cytoplasmic staining was found in 7 cases (Fig. 2D). Normal pancreas was GATA3-negative. Cytoplasmic GFAP staining was negative in all SPN cases (not shown); rare small nerves surrounded by neoplastic tissue were GFAP-positive. Small scattered nerves were the only GFAP-positive structures in the normal pancreas. Membranous/cytoplasmic NCAM/CD56 was detected in all SPN cases (Fig. 2E); 90-100\% of cells showed moderate/strong staining. Two cases showed some heterogeneity in stain intensity across the cores. Nerves in SPN and in normal pancreas were strongly NCAM-positive. Normal pancreas showed NCAM expression in islets, periacinar spindle structures (possibly axons, Schwann cells, or pancreatic stellate cells, PSC), and centroacinar-cell/intercalated-duct compartment. However, expression in the latter two areas differed in extent and intensity across the cores. Cytoplasmic NF was found in $3(19 \%)$ SPN cases, which showed weak granular cytoplasmic staining in 5-10\% of cells (Fig. 2F); other SPN were non-reactive. Nerves were well decorated in both SPN and normal pancreas. Exocrine/endocrine pancreas was NF-negative; rare spindle periacinar structures (possibly axons) were positive. Cytoplasmic S100 staining was detected in 10 (62\%) SPN cases; the range of immuno-positive cells was from $1 \%$ up to $80 \%$ (median $5 \%$ of cells). Staining was usually weak and focal; in some other cases diffusion of the chromogen from S100-positive nerves was evident. In a recurrent case the extent and intensity of the cytoplasmic stain were similar to the primary lesion. In a single case with histopathological features of malignancy the cytoplasmic stain in $80 \%$ of cells was accompanied with nuclear stain in approximately $5 \%$ of cells (Fig. 2G). In normal pancreas, S100 stain was detected in nerves, numerous spindle periacinar structures as well as some islet cells; a portion of islet cells showed not only cytoplasmic but also nuclear S100 expression. Cytoplasmic SYP was found in tumoral cells in 14 (88\%) SPN cases, and its intensity in immune-positive cases ranged from weak to strong (Fig. $2 \mathrm{H}$ ); the extent ranged from $5 \%$ to $90 \%$ of cells. A single recurrent lesions showed more intense (moderate vs. weak) and more diffuse (60\% vs. $40 \%)$ expression in comparison to the primary lesion. In normal pancreas SYP expression was evident in pancreatic islets and numerous spindle periacinar structures, possibly axons and/or PSC. Nerves were positive in both SPN and normal pancreas.

\section{Results of IHC examinations in the validation cohort - SPN}

Results are summarized in Table II. Twelve SPN cases were included. Their clinico-pathological characteristics were previously described $[2,31]$. Ten cases were available for L1CAM/NGFR assessment due to tissue exhaustion in the block. Membranous and cytoplasmic L1CAM was found in $90 \%$ of cases; stain extent in immuno-positive cases ranged from 5\% to $100 \%$; staining was weak/moderate in intensity. NGFR was detected in all 10 SPN cases, stain extent ranged from $10 \%$ to $100 \%$, staining was weak (5 cases), moderate (3 cases), or strong (2 cases). Distribution of L1CAM and NGFR expression in the normal pancreas was the same as in the training cohort.

\section{Results of IHC examinations in the validation cohort - NET}

Results are summarized in Table II. Clinico-pathological data were described in the previous paper [31]. Among 29 included NET cases (Fig. 1D), 27 cases were L1CAM-negative (Fig. E). L1CAM was found in 2 cases only (7\%) - a single case showed heterogeneous staining in $80 \%$ of cells (from weak to strong, mainly weak), and another case showed weak staining in $10 \%$ of cells. NGFR was almost always negative (Fig. 1F) - expression was found in $1(3 \%)$ case only - heterogeneous staining (mainly moderate but focally strong) was found in $70 \%$ of cells. The IHC profile and histopathological picture of L1CAM/NGFR-positive NET cases were not compatible with diagnosis of SPN or paraganglioma. Importantly, stromal cells were NGFR-positive in 26 (90\%) NET cases - focally (12 cases) or diffusely (14 cases). Distribution of L1CAM and NGFR expression in the normal pancreas was the same as in the SPN cohort.

\section{Diagnostic performance of L1CAM/NGFR stains}

L1CAM/NGFR histoscores in the training cohort were not correlated with themselves or with the patients' 
age, tumor diameter, presence of clinical/histopathological malignancy, or histoscores of other IHC markers tested. L1CAM/NGFR/SYP [2] histoscores examined in the whole SPN population $(n=26)$ were not correlated either. Diagnostic performance of L1CAM/NGFR in detection of SPN vs. NET was described in detail in Supplementary Data 3 and Supplementary Data 4. Generally speaking, both markers performed well: (1) any level of L1CAM staining was sensitive for SPN detection (90\%), (2) at least moderate L1CAM staining was fully specific for SPN (100\%), (3) any NGFR positivity was highly sensitive (100\%) and specific (96.6\%) for SPN.

\section{Discussion}

There were several findings of the study: (1) SPN were frequently L1CAM-positive, but its expression levels were usually low/weak, (2) NGFR was present in all SPN cases, and expression was usually moderate/strong, (3) L1CAM/NGFR IHC stains may be supportive in differential diagnosis of SPN vs. NET, with some important limitations (as discussed below), (4) SPN frequently expressed ENO2/NSE, NCAM/CD56, SYP and cytoplasmic S100 (focally), (5) B3GAT1/CD57, NF, and nuclear S100 expression in SPN was rare, (6) SPN did not stain with CHGA, (nuclear) GATA3, and GFAP antibodies, (7) SPN expressed some neural/neuroendocrine/neuroectodermal/NC markers, and the extent and intensity of their expression differed between particular SPN cases, (8) the IHC profile of SPN may support its origin from NC.

\section{Differential diagnosis of SPN vs. NET}

The prognosis for patients with SPN is usually favorable, in contrast to NET, which may progress/ recur at significant frequency $[11,12]$. Therapeutic strategies in SPN and NET are different [35]. However, macro-/microscopic features of SPN and NET may be similar $[1,11,12]$. A pitfall of IHC examinations for SPN vs. NET distinction comes from the fact that SPN may express neuroendocrine markers (especially NCAM/CD56, ENO2/NSE, or SYP) $[1,11,12]$.

\section{Histogenesis of SPN}

The histogenesis of SPN is uncertain $[1,5,25]$. It was hypothesized that SPN may evolve from NC [5, 25]. NC cells are involved in interaction with pancreatic epithelium and in development of $\beta$-cells during pancreatic organogenesis [36]. They subsequently transform into neural and glial cells of the pancreas [36]. Neurotrophin signaling and cell adhesion pathways may be involved in the pathogenesis of SPN [9]. Some signaling pathways (endothelin-3 pathway)

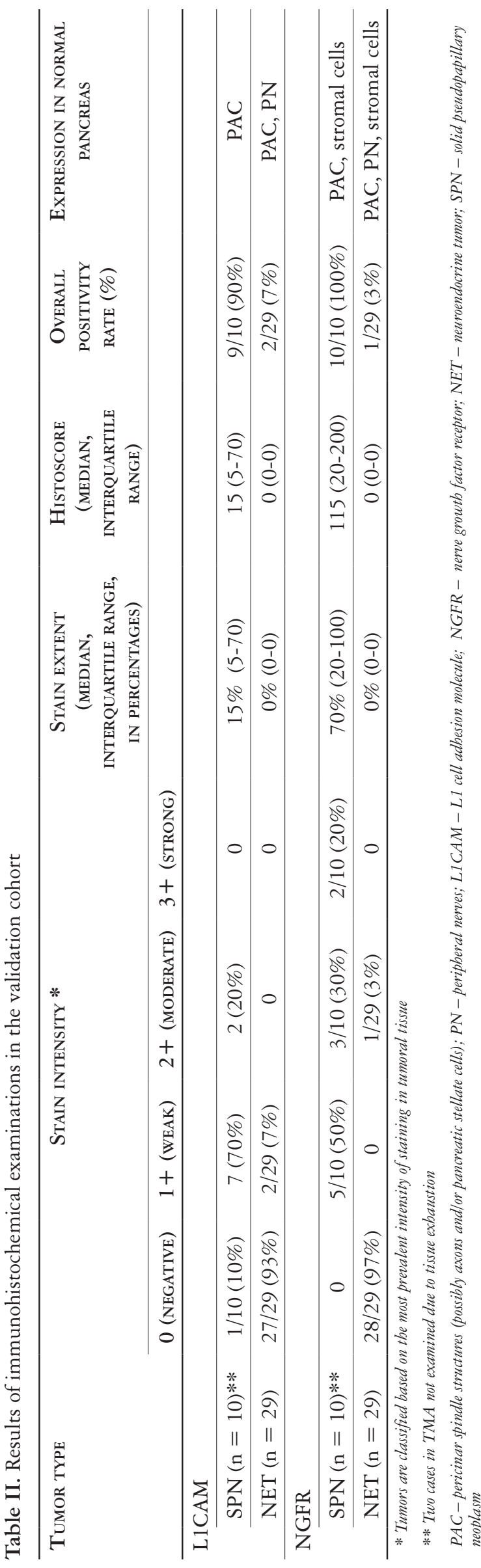


or molecules (SOX10, HAND2, netrins) are important both for the enteric system [37] and SPN [5]. SPN may express some (SOX10, S100), but not all (GFAP) enteric glial markers, or some (TUBB3, SYP) enteric neuron markers [5].

\section{L1CAM}

L1CAM is expressed in postmitotic neurons in the central and peripheral nervous system and in non-myelinating Schwann cells [17]. L1CAM expression is frequent in neoplasms derived from $\mathrm{NC} /$ neuroectoderm, e.g. neuroblastomas, granular cell tumors, phaeochromocytomas, schwannomas, primitive neuroectodermal tumors, and paragangliomas (up to $100 \%$ in each entity) $[27,28,29]$. Melanocytic, mesenchymal, epithelial, and even hematologic tumors may also be L1CAM-positive, albeit usually at lower frequency $[27,28,29]$.

L1CAM was identified as a target gene of the $\beta$-catenin/Wnt signaling pathway [38]. LEF1, which is a marker of SPN [14], binds to the L1CAM promoter [38]. L1CAM may also serve as a potential therapy target in tumors [17].

Expression patterns of L1CAM documented in the pancreas and pancreatic diseases differ somewhat between reports, and this is partially related to the clone of antibody utilized in particular studies. However, both exocrine and endocrine components of the normal pancreas are L1CAM-negative [27,28]. L1CAM was usually detected in ductal adenocarcinomas [39]. Inaguma et al. found L1CAM expression in the majority of NET of the pancreas, but the frequency and intensity of staining was related to the clone of antibody used in the analysis: weak and strong expression was found in $50 \%$ and $23.8 \%$ of cases (respectively) examined with both 014 and L1-14.10 antibodies, but in $42.9 \%$ and $9.5 \%$ of cases (respectively) examined with UJ127.11 antibody [29]. Almost half of the cases $(47.6 \% ; 20 / 42)$ were negative with UJ127.11 antibody [29]. Rawnaq et al. found strong L1CAM expression (UJ127) in 8\% out of 63 "pancreatic neuroendocrine carcinomas" [27]. Kaifi et al. found L1CAM (UJ127) in 1/54 (1.9\%) pancreatic well-differentiated NET [40]. Huszar et al. did not detect L1CAM (L1-14.10 and L1-11A) in 10 "neuroendocrine carcinomas" of the pancreas, or in pancreatic islets [28]. The differences between studies related to prevalence of L1CAM expression may be caused by experimental conditions, scoring criteria, and population bias [39]. In particular, the clone of IHC antibody may influence results. Antibodies differ in regard to their corresponding epitopes in L1CAM protein, e.g. the epitope for the UJ127 antibody (used in this study) is located in the extracellular domain within the fibronectin type III repeats region [16]. L1-14.10 and L1-11A (subclone of UJ127.11) antibodies give comparable
[28], but not always the same [29], results of staining across normal or neoplastic tissues.

In this study L1CAM expression was an almost universal feature of SPN, but expression levels were usually weak. This will probably limit the potential usefulness of this stain for SPN diagnosis. In contrast to SPN, L1CAM was rare in NET in this study (7\%), but importantly some investigators observed its expression in more than $50 \%$ of NET [29]. Technical details of IHC assay may influence the diagnostic performance of L1CAM for SPN vs. NET differential diagnosis. Moreover, some NET may show a paraganglioid pattern of growth, and they may be indistinguishable from paragangliomas without IHC [41]. This is important for interpretation of L1CAM IHC in NET, since paragangliomas are L1CAM-positive in up to $97 \%$ of cases [29].

\section{NGFR}

Neurotrophins are responsible for maintenance, differentiation and survival of neurons in the central and peripheral nervous system, nerve regeneration, and tumor invasiveness [21, 22, 24]. In normal tissues NGFR is expressed in perineurium, epineurium and endoneurium, axons and ganglion cells, Schwann cells, satellite cells of the autonomic ganglia, intestinal neural plexi, pericytes, perivascular mesenchymal cells and some rare epithelial cells [19, 42, 43]. NGFR may be found in peripheral nerve sheath tumors/lesions (schwannomas, neurofibromas, traumatic neuromas, neurofibromatosis, malignant peripheral nerve sheath tumors, granular cell tumors, some cases of melanoma, phaeochromocytomas, ganglionic cells of ganglioneuromas and ganglioneuroblastomas, some mesenchymal non-neural tumors and rare epithelial tumors [19, 42, 43]. Peritumoral stroma may be NGFR-positive regardless of the histotype of the tumor [43].

NGFR may also promote tumor development and take part in regulation of cancer stem cells [44]. NGFR is a potential therapeutic target in cancer [21].

At the proteomic/IHC level, NGFR is virtually absent in epithelia of normal pancreas [42], or it is expressed weakly in the cytoplasm of rare ductal cells, but not in acinar/islet cells [32]. NGFR expression in ductal adenocarcinoma determined using IHC was weak/absent [45] or detected in areas of perineural invasion [46]. Data on NGFR in NET are limited. Chesa et al. found NGFR in 1 of 3 "carcinoid tumors", but not in 2 tested islet cell tumors [42]. Thompson et al. reported partial NGFR expression in 2 out of 3 "carcinoids" [43]. Additionally, NGFR may be a marker of a subset of sustentacular cells in pulmonary carcinoids [47].

In the present study NGFR performed successfully as an SPN marker. It was detected in all examined SPN cases, usually at moderate/high expression levels. 
This contrasted with NET, which were NGFRnegative, with the exception of one case only. This makes NGFR potentially useful as a marker for SPN vs. NET differential diagnosis. However, some related issues should be emphasized: (1) NGFR positivity did not exclude NET diagnosis, (2) both SPN and NET frequently showed NGFR expression in the tumoral stroma. The latter may result in false-positive diagnosis of SPN, as nonspecific diffusion of chromogen from the stroma to the neoplastic cells may mimic true expression in tumoral cells.

\section{NGFR, pancreatic stellate cells (PSC), and SPN}

PSC are functional stromal-myofibroblastic cells of the pancreas, responsible for phagocytosis, immunity, and control of exocrine secretion [48]. Interestingly, it was found that NGFR is a marker of PSC $[45,49]$. Neuroectodermal origin of PSC was considered [49]. However, hepatic stellate cells, which are similar phenotypically to PSC [49], are of mesenchymal rather than of NC origin [50]. One may speculate that the phenotypic profile of SPN may resemble PSC, to some extent. Other PSC markers (e.g. MME/ CD10, nestin, SYP, vimentin) may also be expressed in SPN [11, 12, 25].

\section{Other neural/neuroendocrine/neuroectodermal/ NC markers in SPN}

ENO2/NSE and NCAM/CD56 were found in all SPN cases in this study, in concordance with previous data [25]. Prevalence of SYP positivity was $88 \%$, and thisvalue washighincomparison tootherstudies, which reported SYP positivity in up to $70 \%$ of cases [13]. In this study it was found that the extent/intensity of SYP expression (and therefore level of neural/ neuroendocrine differentiation in SPN) differed between particular cases. In contrast, all SPN were CHGA-negative, in agreement with the literature data [13]. Similarly, we confirmed lack of GFAP in SPN [5]. GATA3 was also negative in all SPN cases, which largely excluded paraganglioid differentiation of SPN. Weak NF was found in 19\% of cases - in one previous study SPN were NF-negative [5]. Focal S100 was found in the majority of cases, but nuclear expression was exceptional. In previous investigations prevalence of S100 expression ranged from nil [5] to $85 \%$ [25]. NF and S100 expression suggests partial neural differentiation in a subset of SPN. The new observation was weak expression of the Schwann cell marker B3GAT1/CD57 in 31\% of SPN. In this study significant heterogeneity concerning some IHC profiles (in particular L1CAM, NGFR, B3GAT1/CD57, NF, S100, SYP) was found across SPN cases. This indicated that SPN is phenotypically heterogeneous disease, in contrast to its largely homogeneous genomic/exomic profile [7].

\section{Limitations of the study}

There were several limitations of the study: (1) The number of cases included in the study was not large. However, SPN is a rare condition. (2) The study was based on TMA samples, and this approach has well-known advantages and limitations. (3) The slides were scored manually by a single observer. Manual assessment of IHC slides by a single individual is still a gold standard in the diagnostic pathology setting. (4) The study was based on tissue resources of a single institution. Further examinations are needed to confirm the results in an independent setting. (5) Some SPN in the validation cohort were somewhat older cases, which might have resulted in diminished intensities/extent of IHC stains. (6) Results of the study may be further confirmed at the mRNA level, or in an IHC study using L1CAM antibody specific to the cytoplasmic domain. (7) Follow-up data were not available for this study.

\section{Conclusions}

L1CAM and NGFR were expressed in SPN, but the intensities and extent of IHC staining differed across the cases. L1CAM and NGFR expression was rare in NET of the pancreas. Therefore both markers may be further tested for their diagnostic utility for SPN vs. NET differential diagnosis in an independent setting. L1CAM and NGFR expression supports previously postulated $\mathrm{NC}$ origin/differentiation of SPN of the pancreas.

The author declares no conflict of interest.

\section{References}

1. Kloppel G, Basturk O, Klimstra DS, et al. Solid pseudopapillary neoplasm of the pancreas. In: WHO classification of tumours. Digestive system tumours. WHO classification of tumours Editorial Board (eds). 5th ed. International Agency for Research on Cancer, Lyon 2019; 340-342.

2. Liszka L, Mrowiec S, Pająk J, et al. Limited usefulness of histopathological features in identification of a clinically aggressive solid-pseudopapillary neoplasm of the pancreas. Pol J Pathol 2014; 65: 182-193

3. Abraham SC, Klimstra DS, Wilentz RE, et al. Solid-pseudopapillary tumors of the pancreas are genetically distinct from pancreatic ductal adenocarcinomas and almost always harbor $\beta$-catenin mutations. Am J Pathol 2002; 160: 1361-1369.

4. Selenica P, Raj N, Kumar R, et al. Solid pseudopapillary neoplasms of the pancreas are dependent on the Wnt pathway. Mol Oncol 2019; 13: 1684-1692.

5. Cavard C, Audebourg A, Letoumeur F, et al. Gene expression profiling provides insights into the pathways involved in solid pseudopapillary neoplasm of the pancreas. J Pathol 2009; 218; 201-209.

6. Guo M, Luo G, Jin K, et al. Somatic genetic variation in solid pseudopapillary tumor of the pancreas by whole exome sequencing. Int J Mol Sci 2017; 18: 81 
7. Wu J, Jiao Y, Dal Molin M, et al. Whole-exome sequencing of neoplastic cysts of the pancreas reveals recurrent mutations in components of ubiquitin-dependent pathways. Proc Nat Asad Sci U S A 2011; 108: 21188-21193.

8. Park M, Kim M, Hwang D, et al. Characterization of gene expression and activated signaling pathways in solid-pseudopapillary neoplasm of the pancreas. Mod Pathol 2014; 27: 580-593.

9. Zhu Y, Xu H, Chen H, et al. Proteomic analysis of solid pseudopapillary tumor of the pancreas reveals dysfunction of endoplasmic reticulum processing pathway. Mol Cell Proteomics 2014; 13: 2593-2603.

10. Park M, Lim JS, Lee HJ, et al. Distinct protein expression profiles of solid-pseudopapillary neoplasms of the pancreas. J Proteome Res 2015; 14: 3007-3014.

11. Notohara K, Wani Y, Fujisawa M. Solid pseudopapillary neoplasm: pathological diagnosis and distinction from other solid cellular tumours of the pancreas. Diagn Histopathol 2008; 14: 266-274.

12. Ohara Y, Oda T, Hashimoto S, et al. Pancreatic neuroendocrine tumor and solid-pseudopapillary neoplasm: key immunohistochemical profiles for differential diagnosis. World J Gastroenterol 2016; 22: 8596-8604.

13. Serra S, Chetty R. Revision 2: an immunohistochemical approach and evaluation of solid pseudopapillary tumour of the pancreas. J Clin Pathol 2008; 61: 1153-1159.

14. Kim EK, Jang M, Park M, et al. LEF1, TFE3, and AR are putative diagnostic markers of solid pseudopapillary neoplasms. Oncotarget 2017; 8: 93404-93414.

15. Harrison G, Hemmerich A, Guy c, et al. Overexpression of SOX11 and TFE3 in solid-pseudopapillary neoplasms of the pancreas. Am J Clin Pathol 2018; 149; 67-75.

16. van der Maten M, Reijnen C, Pijnenborg JMA, et al. L1 cell adhesion molecule in cancer, a systematic review on domain-specific functions. Int J Mol Sci 2019; 20: 4180.

17. Gavert N, Ben-Shmuel A, Raveh S, et al. L1-CAM in cancerous tissues. Expert Opin Biol Ther 2008; 8: 1749-1757.

18. Hua T, Liu S, Xin X, et al. Prognostic significance of L1 cell adhesion molecule in cancer patients: a systematic review and meta-analysis. Oncotarget 2016; 7: 851960-85207.

19. Fanburg-Smith JC, Miettinen M. Low-affinity nerve growth factor receptor (p75) in dermatofibrosarcoma protuberans and other nonneural tumors: a study of 1,150 tumors and fetal and adult normal tissues. Hum Pathol 2001; 32: 976-983.

20. Hempstead BL. The many faces of p $75^{\text {NTR }}$. Curr Opinion Neurobiol 2002; 12: 260-267.

21. Blondy S, Christou N, David V, et al. Neurotrophins and their involvement in digestive cancers. Cell Death Dis 2019; 10: 123

22. Schor NF. The p75 neurotrophin receptor in human development and disease. Progress Neurobiol 2005; 77: 201-214.

23. Le Douarin NM, Kalcheim C. The neural crest. Second edition. Cambridge University Press, Cambridge 1999.

24. Wislet S, Vandervelden G, Rogister B. From neural crest development to cancer andvice versa: how $\mathrm{p} 75^{\mathrm{NTR}}$ and (pro)neurotrophins could act on cell migration and invasion? Fron Mol Neurosci 2018; 11: 244.

25. Ye J, Ma M, Cheng D, et al. Solid-pseudopapillary tumor of the pancreas: clinical features, pathological characteristics, and origin. J Surg Oncol 2012; 106: 728-735.

26. Anderson RB, Turner KN, Nikonenko AG, et al. The cell adhesion molecule L1 is required for chain migration of neural crest cells in the developing mouse gut. Gastroenterology 2006; 130: 1221-1232.

27. Rawnaq T, Quaas A, Zander H, et al. L1 is highly expressed in tumors of the nervous system: a study of over 8000 human tissues. J Surg Res 2012: 173: 314-319.

28. Huszar M, Moldenhauer G, Gschwend V, et al. Expression profile analysis in multiple human tumors identifies L1 (CD171) as a molecular marker for differential diagnosis and targeted therapy. Hum Pathol 2006; 37: 1000-1008.

29. Inaguma S, Wang Z, Lasota JP, et al. Expression of neural cell adhesion molecule L1 (CD171) in neuroectodermal and other tumors: an immunohistochemical study of 5155 tumors and critical evaluation of CD171 prognostic value in gastrointestinal stromal tumors. Oncotarget 2016; 7: 55276-55289.

30. Hoshi N, Hiraki H, Yamaki T, et al. Frequent expression of $75 \mathrm{kDA}$ nerve growth factor receptor and phosphotyrosine in human peripehral nerve tumours: an immunohistochemical study on paraffin-embedded tissues. Virchows Arch 1994; 424: 563-568.

31. Liszka E. Alpha-methylacyl-CoA racemase is expressed in a majority of pancreatic neoplasms of neuroendocrine, acinar, and solid pseudopapillary differentiation. Pathology 2015; 47 : 466-468.

32. Zhu Z, Firess H, Shi X, et al. Up-regulation of p75 neurotrophin receptor (p75NTR) is associated with apoptosis in chronic pancreatitis. Dig Dis Sci 2003; 48: 717-725.

33. Abramson JH. WINPEPI updated: computer programs for epidemiologists, and their teaching potential. Epidemiol Perspect Innov 2011; 8: 1

34. Knijn N, Nagtegaal ID. Guidelines for reporting histopathology studies. J Clin Pathol 2015; 68: 173-174.

35. Meriden Z, Shi C, Edil BH, et al. Hyaline globules in neuroendocrine and solid-pseudopapillary neoplasms of the pancreas: a clue to the diagnosis. Am J Surg Pathol 2011; 35: 981-988.

36. Nekrep N, Wang J, Miyatsuka T, et al. Signals from the neural crest regulate beta-cell mass in the pancreas. Development 2008; 135: 2151-2160.

37. Barlow AJ. Neural crest cells in enteric nervous system devlopment and disease. In: Trainor PA (ed.) Neural crest cells. Evolution, development and disease. Elsevier, London 2014, pp. 231-254.

38. Gavert N, Conacci-Sorrell M, Gast D, et al. L1, a novel target of beta-catenin signaling, transforms cells and is expressed at the invasive front of colon cancers. J Cell Biol 2005; 168: 633-642.

39. Bergmann F, Wandschneider F, Sipos B, et al. Elevated L1CAM expression in precursor lesions and primary and metastatic tissues of pancreatic ductal adenocarcinoma. Oncol Rep 2010; 24: 909-915.

40. Kaifi JT, Zinnkann U, Yekebas EF, et al. L1 is a potential marker for poorly-differentiated pancreatic neuroendocrine carcinoma. World J Gastroenterol 2006; 12: 94-98.

41. Xue Y, Reid MD, Pehlivanoglu B, et al. Morphologic variants of pancreatic neuroendocrine tumors: clinicopathologic analysis and prognostic stratification. Endocr Pathol 2020, 31: 239253.

42. Chesa PG, Rettig WJ, Thomson TM, et al. Immunohistochemical analysis of nerve growth factor receptor expression in normal and malignant human tissues. J Histochem Cytochem 1988; 36: 383-389.

43. Thompson SJ, Schatteman GC, Gown AM, et al. A monoclonal antibody against nerve growth factor receptor. Immunohistochemical analysis of normal and neoplastic human tissue. Am J Clin Pathol 1989; 92: 415-423.

44. Chopin V, Lagadec C, Toillon RA, et al. Neurotrophin signaling in cancer stem cells. Cell Mol Life Sci 2016; 73: 18591870.

45. Fujiwara K, Ohuchida K, Mizumoto K, et al. CD271+ subpopulation of pancreatic stellate cells correlates with prognosis of pancreatic cancer and is regulated by interaction with cancer cells. PloS ONE 2012; 7: e52682.

46. Wang W, Zhao H, Zhang S, et al. Patterns of expression of and function of the $\mathrm{p} 75^{\mathrm{NGFR}}$ protein in pancreatic cancer cells and tumours. Eur J Surg Oncol 2009; 35: 826-832.

47. Gosney JR, Denley H, Resl M. Sustentacular cells in pulmonary neuroendocrine tumors. Histopathology 1999; 34: 211-215. 
48. Allam A, Thomsen AR, Gothwal M, et al. Pancreatic stellate cells in pancreatic cancer: in focus. Pancreatology 2017; 17: 514-522.

49. Haeberle L, Steiger K, Schiller AM, et al. Stromal heterogeneity in pancreatic cancer and chronic pancreatitis. Pancreatology 2018; 18: 536-549.

50. Cassiman D, Barlow A, Vander Borght S, et al. Hepatic stellate cells do not derive from the neural crest. J Hepatol 2006; 44: 1098-1104.

\section{Address for correspondence}

\section{Łukasz Liszka}

Department of Pathomorphology and Molecular Diagnostics

Medical University of Silesia,

Katowice, Poland

e-mail: lliszka@mp.pl 